\title{
Long-Term Functional Outcomes of Endoscopic Decompression with Destandau Technique for Lumbar Canal Stenosis
}

\author{
Saransh Gupta ${ }^{1}$, Nandan Marathe ${ }^{1}$, Harvinder Singh Chhabra ${ }^{1}$, Jean Destandau ${ }^{2}$ \\ ${ }^{1}$ Department of Spine Service, Indian Spinal Injuries Centre, New Delhi, India \\ ${ }^{2}$ Department of Neurosurgery, Bel Air Clinic, Bordeaux, France
}

Study Design: Retrospective study of patients with lumbar canal stenosis (LCS) operated using endoscopic unilateral laminotomy with bilateral decompression (ULBD).

Purpose: This study aimed to provide a detailed description of the technique of endoscopic decompression in LCS along with a description of the surgical anatomy and its advantages. We also discuss the clinical outcomes in patients operated using this technique. Overview of Literature: In 1999, the results with the use of microscopic ULBD were published. Microscopic/microendoscopic decompression using tubular retractor system showed good to excellent results in studies that compared such techniques with midline decompression. The first description of the use of endoscope in spine surgery was in 1988 when it was used for discectomy. With advancements and familiarity with the techniques, full endoscopic surgery has found application in LCS treatment.

Methods: The clinical records of 953 patients who were operated between 1998 and 2008 were analyzed in 2018. Along with patient characteristics, information about return to daily activities, complication rates, and functional outcomes using Prolo score was assessed.

Results: L4-L5 was the most common level for which surgery was performed. Two-level decompression was performed in 116 patients; $89.5 \%$ patients were able to return to their daily activities after 2 weeks. Functional outcomes as per the Prolo score were reported by patients as excellent, good, and poor in $89.85 \%, 1.59 \%$, and $8.55 \%$, respectively. Repeat surgery was required at same level in 16 patients and at a different level in 21 patients. Total 605 patients (63.49\%) were symptom-free during the 70-month followup, while 344 complained of residual back pain, and four complained of persistent leg pain.

Conclusions: ULBD using the Endospine system achieves adequate decompression in most cases and is a good alternative to open laminectomy, with the advantage of avoiding damage to the structural integrity of the spine and preserving soft tissue attachments.

Keywords: Destandau technique; Lumbar canal stenosis; Endoscopic decompression

\section{Introduction}

Lumbar canal stenosis (LCS) is one of the most common spinal pathologies observed in the elderly population. The stenosis is owing to bony, ligamentous, discal, and capsular structures that impinge on to the neural elements. How-

Received Mar 22, 2020; Revised Jul 8, 2020; Accepted Aug 1, 2020

Corresponding author: Nandan Marathe

Department of Orthopaedics, Seth GS Medical College and KEM Hospital, Saraswati Prasad, Gaul Wada, Vasai (West), Palghar, Maharashtra, 401201, India

Tel: +91-0250-2309655, Fax: +91-6476795733, E-mail: drnandanmarathe@kem.edu 
ever, there is no unequivocal evidence to suggest that the amount of compression visualized in imaging studies correlates directly with symptom severity [1]. Cases with the intolerable persistence of symptoms or progressive deterioration of functional ability warrant surgical intervention. There is an increase in the number of decompression surgeries for spinal stenosis [2]. The conventional approach is decompression with open laminectomy that involves stripping of the paraspinal muscles on both sides of the spinous process and removal of the midline structures and the lamina. Concerns have been raised about the invasiveness of these conventional open procedures $[3,4]$. The long retraction of the paraspinal muscles and the disturbed blood supply results in muscle atrophy and damage to the dorsal rami, posterior tension band, and altered biomechanics of the posterior ligamentous structures can result in persistent back pain and instability [5-7].

In recent years, there has been a trend to move away from more aggressive techniques and adopt minimally invasive methods. These techniques are used with the objective of reducing the tissue damage and improving the clinical outcomes $[8,9]$. The goal of any spine decompression surgery is the achievement of adequate decompression in a safe manner without damage to the normal structures. An important consideration in the development of new techniques is the achievement of better visualization, magnification, and illumination, with minimal tissue trauma. Endoscopic spine surgery is a form of minimally invasive technique that has been described widely in lumbar and cervical discectomy. The goal of the present study was to examine the results and technical feasibility with endoscopic unilateral laminotomy with bilateral decompression (ULBD) using the Endospine system or Destandau's technique. We shall discuss the surgical steps and technical details in this manuscript.

\section{Materials and Methods}

The medical records of patients with spinal stenosis operated using the Endospine technique were retrospectively reviewed. Patients operated between 1998 and 2008 were enrolled. These cases were analyzed in 2018 to ensure a minimum 10-year follow-up for every patient. Written informed consent was obtained from all the patients included in the study for sharing of their clinical data. As per the inclusion criteria, patients with a diagnosis of degenerative LCS with complaints of axial back pain and neurogenic claudication who were refractory to conservative measures for $>3$ months were included. Conservative measures included medical management (analgesics) and epidural injections (steroid with local anesthetic agent). Patients with spondylolisthesis >grade II were excluded. Total 953 patients satisfied the inclusion criteria and were enrolled. Approval was obtained from the institutional review board of Bel Air Clinic, Bordeaux, France before initiating the study (IRB approval no., IRB/BAC/1997-23).

All the patients were clinically examined by the same surgeon who is also the lead author of this manuscript; thus, there was no risk of bias. Radiological investigations that were performed included radiography (anteriorposterior, lateral radiographs in neutral, flexion, and extension), magnetic resonance imaging (MRI), computed tomography (CT) scan, and myelogram. CT scan was performed during the initial years owing to the ease of availability in near the institute of work; myelogram was added in case the CT scan did not reveal the compression site adequately. Then, with the advent of MRI, it was used as the imaging modality of choice.

\section{Operative technique}

The operative technique is described in a logical sequence of steps that includes patient positioning, room set up, marking of the level, setting up the lighting, posterior spinal exposure, endoscopic decompression, and closure. The surgery can be performed under general anesthesia or spinal anesthesia. All the patients in our series were operated by a single surgeon who is also the lead author of this paper.

\section{Patient position}

The patient was positioned in a kneeling prone position. Not only does it help in opening the interlaminar window, but also allows abdomen to hang free and minimizes the engorgement of the lumbar epidural veins. The lumbar spine is maintained in the neutral position or with some amount of lordosis (Fig. 1). The neck is turned to the side, and the arm is positioned over the side arm rest. Care must be exercised to avoid peri-orbital pressure and ulnar nerve impingement. The hips are flexed to around $100^{\circ}$ and abducted around $30^{\circ}$. This helps in achieving a stable position. The knees are supported on the sides by cushioned side supports. 


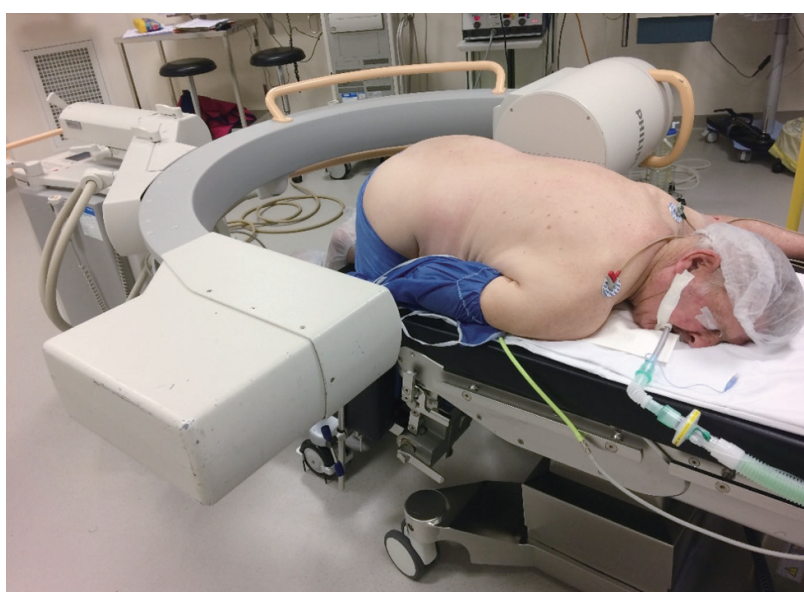

Fig. 1. Positioning of the patient. Written informed consent for publication of this image was obtained from the patient.

\section{Instruments}

Standard arthroscopic facilities and conventional spine instruments, such as Kerrison rongeurs $\left(45^{\circ}\right.$ and $\left.90^{\circ}\right)$, pituitary forceps, Endospine system, and high-speed burr are needed.

\section{Operating room set up}

The surgeon stands on the side of the approach, and the assistant stands on the opposite side. The Mayo trolley with instruments is on the left side of the surgeon. This helps keep the suction and the endoscope camera tubings away from the instrument port and helps keep the Endospine system stable. The monitor is placed in front of the operating surgeon.

\section{Localization and setting up the light}

A preliminary fluoroscopy image focusing over the target level in the lateral position is taken, and C-arm is positioned to focus the target level at the center of the image. A specially designed metallic marker tool with two side arms is placed over the back of the patient, and the side arms are adjusted to superimpose over each other and fall in line with the disc space (Fig. 2). Once the correct positioning is achieved, the skin is marked with the surgical marking pen. Lighting is adjusted to focus over the level marked, parallel and in line with the side arms of the metallic marker tool. This helps in navigating the surgeon during exposure to reach the right level.

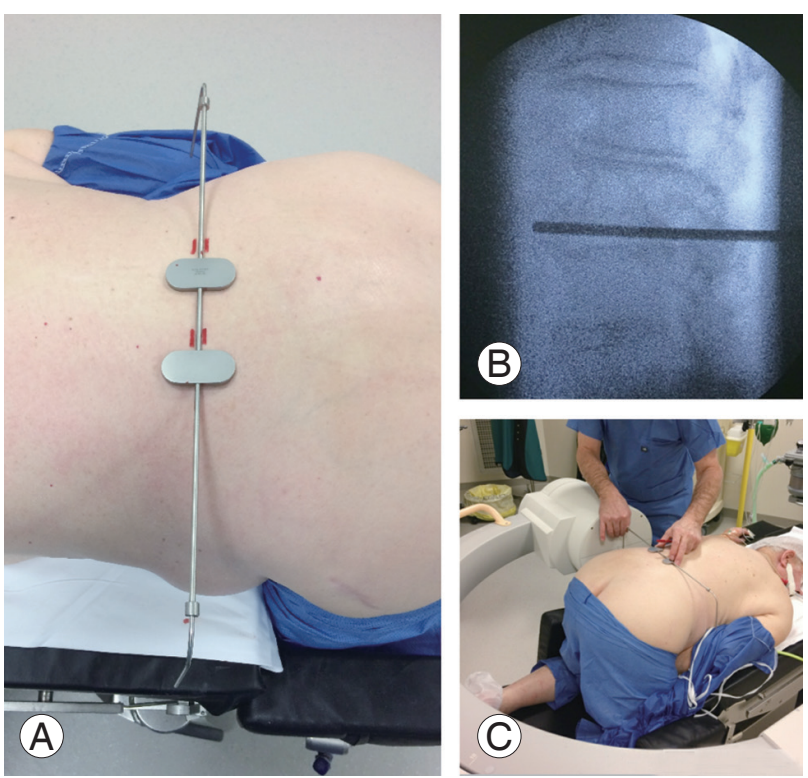

Fig. 2. (A-C) Confirmation of the level to be operated on $C$ arm. Written informed consent for publication of this image was obtained from the patient.

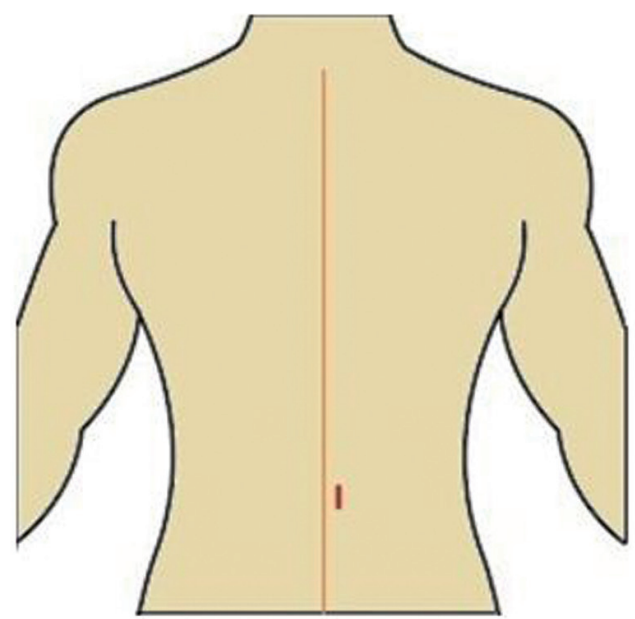

Fig. 3. Longitudinal skin incision is made about $1 \mathrm{~cm}$ lateral to the spinous process.

\section{Posterior spinal exposure}

A longitudinal skin incision is made about $1 \mathrm{~cm}$ lateral to the spinous process (Fig. 3). An incision is made deep through the dermal layer and subcutaneous fat. Bipolar cautery is used to catch the bleeding points. The paramedian incision helps in angulating the Endospine (Karl Storz, Bordeaux, France) to reach the opposite side. The paraspinal muscle fascia is cut using Mayo scissors and subsequently with a periosteal elevator; the spinal muscles are erased off the spinous process. A Karl Storz metal re- 

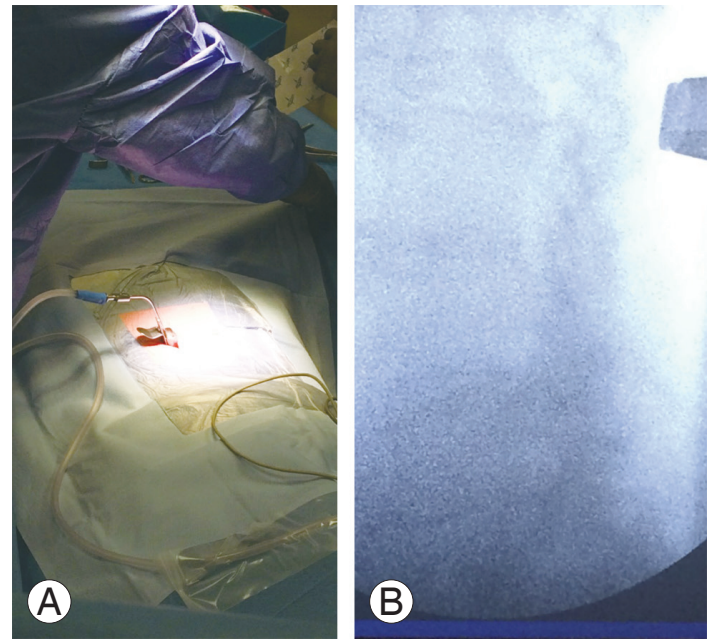

Fig. 4. (A, B) Karl Storz metal retractor with outer sheath of the Endospine with the obturator. tractor is inserted to keep the paraspinal muscles retracted laterally followed by the insertion of the outer sheath of the Endospine with the obturator that is pushed between the retractor laterally and spinous process medially (Fig. 4). The field is cleaned with pituitary forceps, followed by insertion of the inner tube of the Endospine with the endoscope attached. A fluoroscopy image can be taken at this point to confirm the proper level (Fig. 5).

\section{Laminotomy/medial facetectomy/ligamentum fla- vum removal}

The endoscopic field will now show the caudal edge of the cranial lamina, the cranial edge of the caudal lamina, and the interlaminar window in between. The laminotomy is
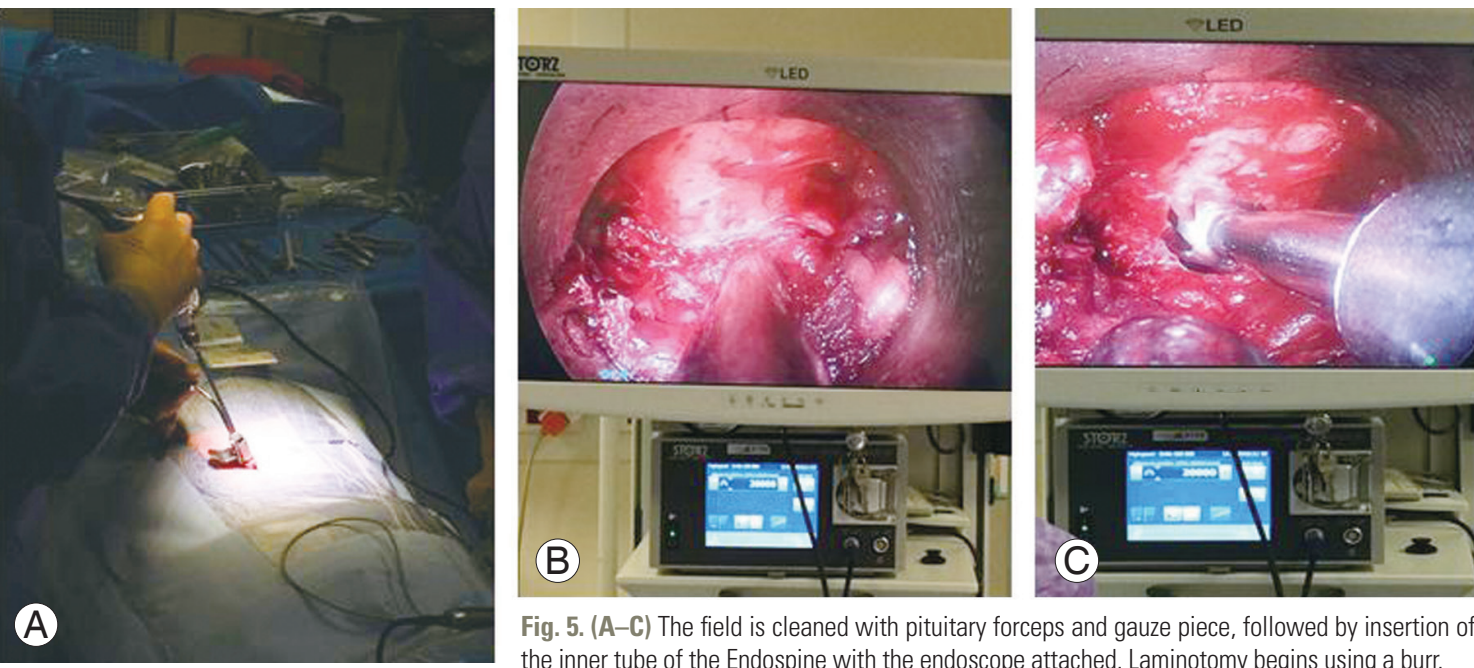

Fig. 5. (A-C) The field is cleaned with pituitary forceps and gauze piece, followed by insertion of the inner tube of the Endospine with the endoscope attached. Laminotomy begins using a burr.
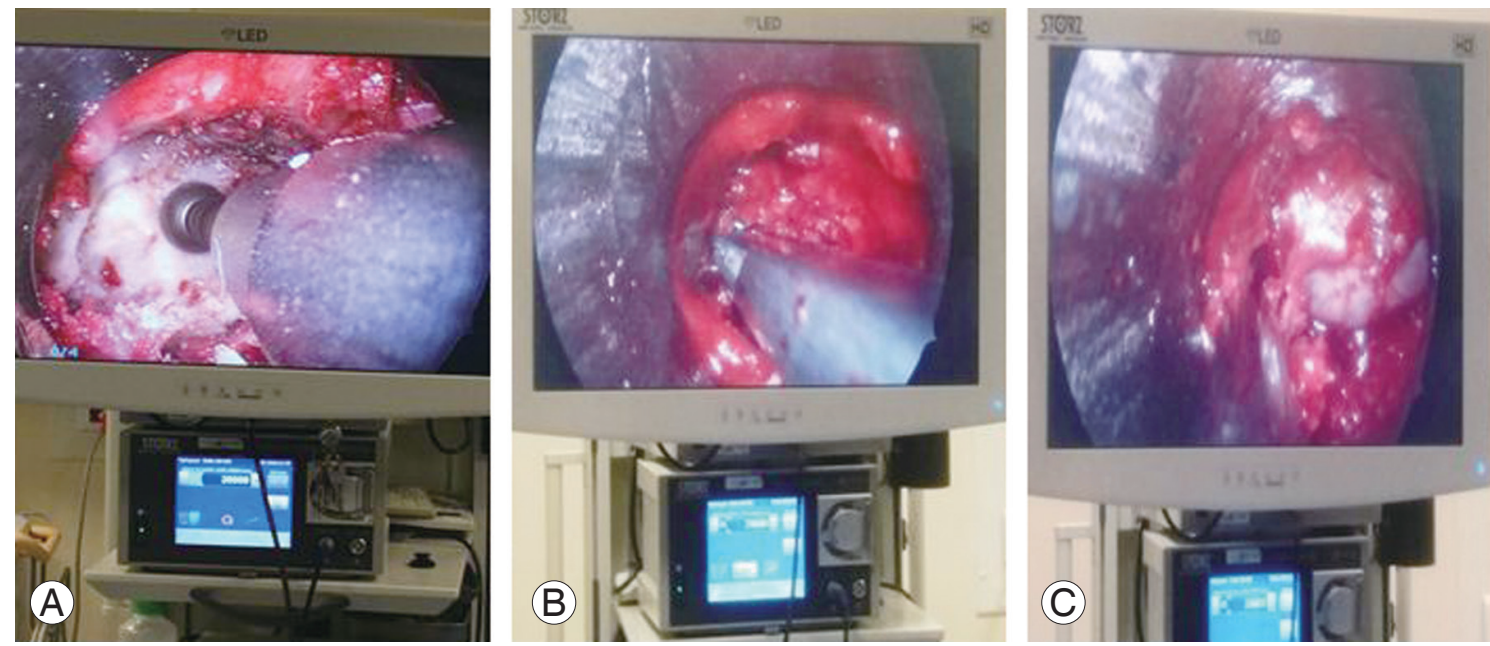

Fig. 6. (A-C) Base of the spinous process is resected with $45^{\circ}$ Kerrison rongeur or endoscopic drill to make room for the Endospine system and working space for carrying out decompression on the opposite side of the midline. 

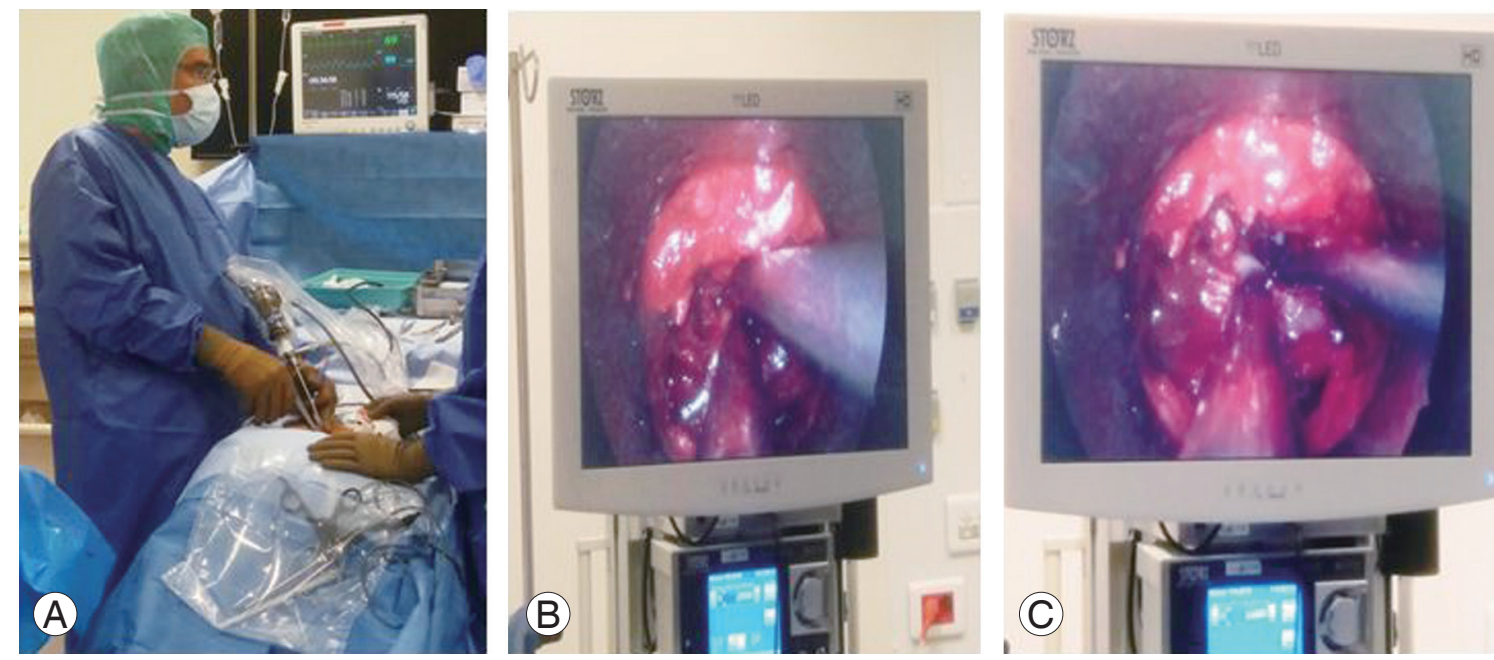

Fig. 7. (A-C) Completion of laminotomy on opposite side.

started through this window (Fig. 6). The lamina of L3, L4, L5 in spinal stenosis patients is often thick; thinning of the lamina to the ventral cortical bone adjacent to the epidural space can be achieved with a high-speed 3-mm tip endoscopic burr (diamond or cutting). Once the caudal edge of the cranial lamina and the cranial edge of the caudal lamina have been thinned, angled Kerrison rongeur can be used to complete the laminotomy piecemeal. A significant portion of the cranial lamina may need to be removed to reach the superior edge of the flavum for its detachment. At this point, dural adhesions may be checked for using a freer elevator or a cottonoid inserted between the flavum and the dura. Laterally, the medial facet is undercut with removal of the flavum to expose lateral edge of the dura and the traversing nerve root. The caudal attachment of the ligament flavum is detached from the cranial part of the caudal lamina and excised using $90^{\circ}$ Kerrison rongeur after checking for the adhesions with a nerve hook or cottonoid. (The ligament flavum can be maintained till the bony work is one on the contralateral side as a protective covering over the dura.)

\section{Over-the-top decompression of the contralateral side}

The base of the spinous process is resected with $45^{\circ}$ Kerrison rongeur or an endoscopic drill to make room for the Endospine system and working space for performing decompression on the opposite side of the midline (Fig. 6). Once adequate space has been created, the Endospine is angulated medially to view the opposite side of the spinal canal and the lateral recess. The ligament is detached off
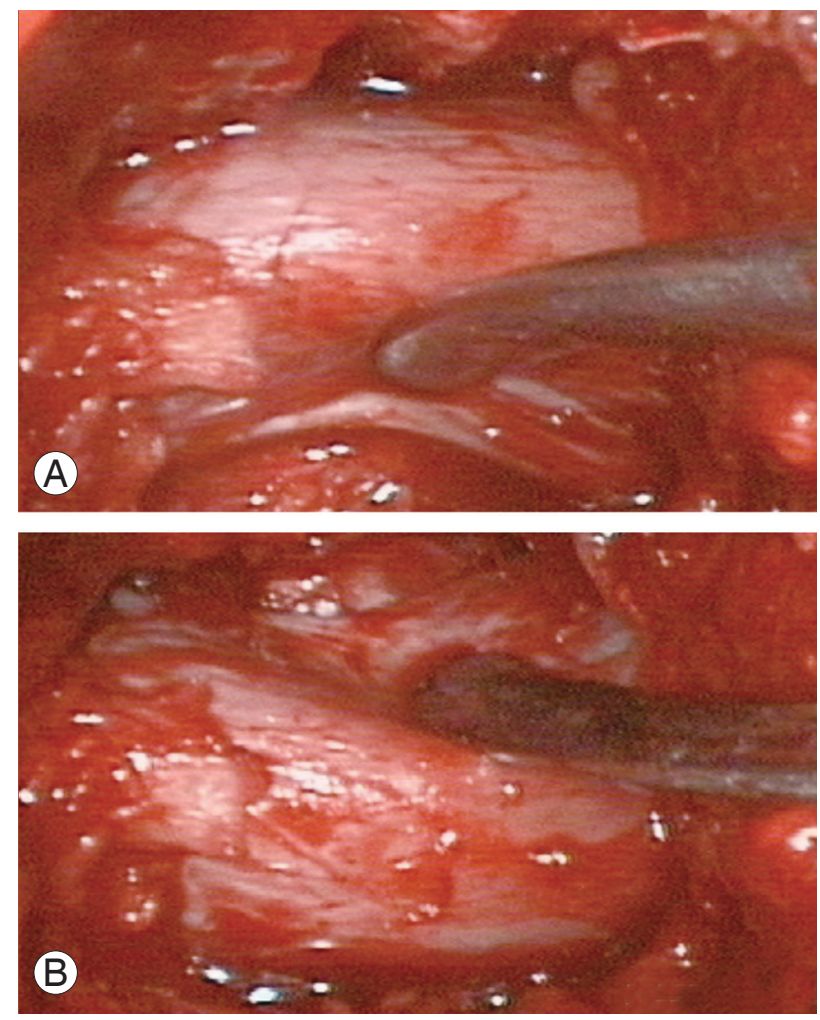

Fig. 8. (A, B) Endpoint of decompression; decompressed thecal sac with ipsilateral and contralateral nerve root.

the cranial and caudal lamina of the opposite side using angled curettes or freer elevator and laminotomy completed using Kerrison or endoscopic drill (Fig. 7). The medial facet on the opposite side is similarly undercut and the flavum is excised with a combination of $90^{\circ}$ Kerrison and pituitary forceps. Cottonoid may be placed over the lateral border of the dura/traversing nerve root and the root 


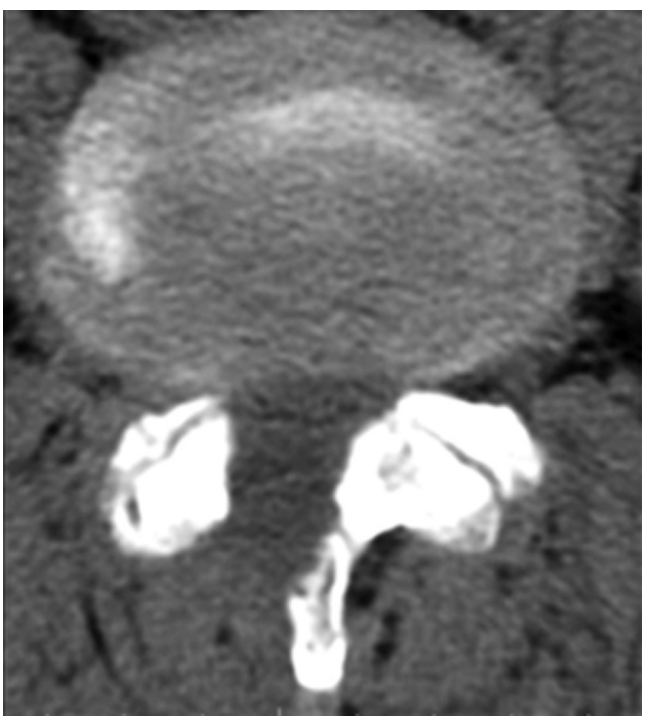

Fig. 9. Postoperative axial computed tomography scan showing the laminotomy and extent of bone removal.

traced to the neural foramen, neural foramen is checked for patency using a Woodson probe which should be able to pass through. The end-point of decompression is when the lateral border of the nerve roots can be visualized bilaterally till the foramen and can be freely mobilized medially (Fig. 8). Endoscopic bipolar is used to achieve hemostasis whenever needed. Once decompression has been achieved, irrigation with saline is done, and the Endospine system is gradually withdrawn. No drain is used. The fascia is closed with Vicryl number 1, and subcutaneous tissue is approximated with inverted sutures using Vicryl 3-0. Postoperative CT scan showed the extent of laminotomy (Fig. 9). In cases of two-level decompression, the authors performed a single skin incision, with two different fascial incisions.

\section{Postoperative care}

The patient is allowed to mobilize without any brace. The patient is usually discharged on postoperative day 2 .

\section{Results}

Since 1998, 953 patients have been operated using the Endospine system with the endoscopic ULBD approach, and a minimum of 10-years follow-up data are available for every patient. Among these, 561 (59\%) were male patients and $392(41 \%)$ were female patients. The average
Table 1. Distribution of lumbar canal stenosis as per the vertebral level

\begin{tabular}{cc} 
Level & No. of patients $(\%)$ \\
\hline L1-L2 & $3(0.31)$ \\
L2-L3 & $60(6.3)$ \\
\hline L3-L4 & $299(31.37)$ \\
L4-L5 & $527(55.3)$ \\
\hline L5-S1 & $45(4.72)$ \\
\hline
\end{tabular}

Table 2. Complications

\begin{tabular}{lc} 
Complication & No. of patients $(\%)$ \\
\hline Dural tear & $64(6.72)$ \\
\hline Facet resection & $30(3.15)$ \\
\hline Nerve root injury & $4(0.42)$ \\
\hline Wrong level surgery & $3(0.31)$ \\
\hline Deep vein thrombosis & $1(0.1)$ \\
\hline
\end{tabular}

patient age was 68 years (range, 24-90 years). The mean delay from the onset of systems till the time of surgery was 111 weeks (range, $0-780$ weeks). Stenosis was found most commonly at the L4-L5 level in 527 patients (55\%). The occurrence of stenosis at different levels is presented in Table 1. Symptoms were predominant on the right side in 173 patients, on the left side in 212 patients, and bilaterally in 559 patients $(18.1 \%, 22.2 \%$, and $58.6 \%$, respectively). Two-level stenosis decompression surgery was performed for 116 patients (12.17\%). There were 103 patients with degenerative spondylolisthesis in our series. However, none of the patients showed progression of the slip on follow-up. Apart from neurogenic claudication, on examination, 142 patients (14.9\%) had motor weakness, 131 (13.75\%) had sensory impairment, and 619 (64.9\%) had abnormal reflexes. Concomitant herniated disc was present in 83 patients $(8.7 \%)$ and associated spondylolisthesis was observed in 165 patients (17.31\%). Radiological investigation included MRI in 659 patients (69.15\%), CT scan in 227 patients (23.82\%), and myelogram in 64 patients $(6.72 \%)$.

The following complications were noted: dual tear in 64 patients $(6.72 \%)$, nerve root injury occurred in four patients $(0.42 \%)$, and deep vein thrombosis in one patient $(0.1 \%)$. Facet resection occurred in 30 patients $(3.15 \%)$; however, this had no effect on the postoperative stability. Wrong level was encountered intraoperatively in three pa- 
Table 3. Post-surgery outcomes

\begin{tabular}{lc} 
Outcome & No. of patients $(\%)$ \\
\hline Excellent & $620(89.85)$ \\
Good & $11(1.59)$ \\
\hline Fair & 0 \\
Poor & $59(8.55)$ \\
\hline
\end{tabular}

tients (0.31\%) (Table 2). Adjacent level degeneration was observed in 3\% of the patients over a 10-year follow-up duration. There was no case of infection in our series.

In our study, 231 patients (24.24\%) were professionally employed preoperatively; of these, 194 (83.98\%) were able to return to their professional work after an average duration of 3 weeks. Further, 344 patients complained of residual back pain, while four had residual leg pain. In all these cases, conservative trial with analgesics was offered initially. Moreover, back-strengthening and core-stabilization exercises were prescribed along with ergonomic advice. If there was no pain relief, imaging was performed again to identify residual compression. Repeat surgery was required at the same level in 16 patients $(1.68 \%)$ and in 21 patients (2.2\%) at a different level with an average interval of 21 months between the two surgeries. Indications for a repeat surgery included recurrent disc herniation, restenosis at the same level, or inadequate pain relief following primary decompression, refractory to conservative management. Repeat surgery was performed by the primary surgeon using the same technique. However, none of the patients in our series required fusion for axial back pain.

A questionnaire for obtaining feedback on functional outcome was distributed to all the patients after 12 months of the surgery. Six hundred and ninety patients responded to the questionnaire satisfactorily. The Prolo score was used for assessing the functional outcomes after the surgery. Total 620 patients $(89.85 \%)$ reported excellent results, 11 (1.59\%) reported good results, and 59 (8.55\%) reported poor results (Table 3). Of these 690 patients, 618 reported being able to get back to their normal life after 2 weeks.

\section{Discussion}

The conventional surgical management of LCS has been open decompressive laminectomy. This has been a very useful procedure; however, it may cause iatrogenic insta- bility $[10,11]$, scarring, and adhesions $[12,13]$ that might require re-intervention. Minimally invasive surgery is gaining wide acceptance and is used in lumbar discectomy, foraminal decompression, spinal fusion, and deformity correction [14]. The advantages of minimally invasive approach include less tissue trauma, blood loss, shorter operative time, lesser hospital stays, and lesser postoperative analgesia requirement.

Biomechanical studies on human cadaver and finite element models have shown that stability of the spine is compromised via the resection of the posterior bony and ligamentous structures [15-18]. Moreover, the crosssectional area of the paraspinal muscles is compromised to a greater extent with open laminectomy than with minimally invasive decompression. In one study, the cross-sectional area decreased by $18 \%$ as compared to that with MISS decompression [19]. Minimally invasive spine surgery with bilateral decompression through the unilateral approach preserves the paraspinal muscle bulk and the posterior tension band and achieves decompression without violating the contralateral facet.

Minimally invasive approaches to LCS include microscopic, microendoscopic, and endoscopic approach. In 1999, Weiner et al. [20] published their results using the technique of microscopic ULBD. They reported excellent results with $87 \%$ of patients reporting high satisfaction rates. Subsequently, other studies reported good to excellent results with microscopic ULBD, including in patient population with grade 1 spondylolisthesis [21,22]. With the advent and use of tubular retractors, the need for muscle stripping became less and the muscle retraction pressure could be reduced, resulting in less crush injury of the muscle [23]. Microscopic and microendoscopic decompression using tubular retractor system showed good to excellent results in studies that compared such techniques with midline decompression $[24,25]$.

The first description of the use of endoscope in spine surgery dates back to 1988 when its use was described for discectomy [26]. With the advancements and familiarity with the techniques, full endoscopic surgery is used in the treatment of LCS. The results have been comparable with those of microscopic decompression $[27,28]$.

The Endospine is elliptical and sits perfectly in the target area between the two laminae in the center, spinous process medially and the medial facet laterally. This is in contrast to the tubular retractor that is cylindrical and is reported to require excessive resection of the medial facet 
to allow it to sit properly and be medialized [24]. The system is not attached to the table and can be maneuvered easily in multiple directions to provide greater freedom to the surgeon and more working area of the instruments. The fixed angle between the suction and the instrument port avoids the intermingling of the two instruments. The surgical outcomes are comparable to those with open and other minimally invasive decompression techniques published in the literature [24,25,27-30].

Acquired LCS is commonly observed in older populations who have multiple co-morbidities. The surgical goal in this age group remains the same; however, there is greater emphasis on minimizing the surgical morbidity and aiding faster recovery during the postoperative phase. Minimally invasive surgery using endoscopic unilateral approach for bilateral decompression is suited to such populations by realizing these important goals. The intraoperative surgical time and blood loss is minimized; postoperative pain is less, and the patient can be mobilized on the same day.

Like in any other minimally invasive technique, there is a learning curve to this approach that may amount to greater surgical time in the beginning and higher chances of complications. Once the learning curve has been cleared and familiarity with this technique has been achieved, it provides excellent visualization, illumination, and access to the site of the pathology.

The article has presented the technique of interlaminar endoscopic LCS ULBD using the Endospine system. This technique has been described for conditions without a significant deformity (high-grade spondylolisthesis/ scoliosis). The procedure has been described in steps that have been supplemented with pictures. The goals and principles have been stressed upon to achieve adequate decompression from side to side as well as cephalad to caudal direction using a safe technique to minimize the chances of dural tear and neurological damage as well as to minimize tissue damage and blood loss. Overall, adequate decompression can be achieved in most cases and is a good alternative to open laminectomy, with the advantage of preventing undue damage to the structural integrity of the spine and preserving the soft tissue attachments. Thus, "minimum invasion maximum relief" can be achieved in cases of LCS using the Endospine system.

The limitations of our study include the retrospective nature of the trial and the fact that it was a single-center single surgeon experience. A prospective multi centric trial is required to further validate our findings. However, the advantage of our series is that it is the largest series with 953 cases and a long follow-up duration for LCS operated using the Endospine system.

\section{Conclusions}

ULBD using Endospine achieves adequate decompression in most cases and is a good alternative to open laminectomy, with the advantage of preventing undue damage to the structural integrity of the spine and preserving soft tissue attachments. Thus, "minimum invasion maximum relief" can be achieved in cases of LCS using the Endospine system.

\section{Conflict of Interest}

No potential conflict of interest relevant to this article was reported.

\section{Author Contributions}

Saransh Gupta: data collection and data analysis; Nandan Marathe: data analysis, editing and proof reading, statistics; Harvinder Singh Chhabra: review of literature; and Jean Destandau: study design and concept.

\section{References}

1. Torgerson WR, Dotter WE. Comparative roentgenographic study of the asymptomatic and symptomatic lumbar spine. J Bone Joint Surg Am 1976;58:850-3.

2. Deyo RA, Mirza SK, Martin BI, Kreuter W, Goodman DC, Jarvik JG. Trends, major medical complications, and charges associated with surgery for lumbar spinal stenosis in older adults. JAMA 2010;303:125965.

3. Thome C, Zevgaridis D, Leheta O, et al. Outcome after less-invasive decompression of lumbar spinal stenosis: a randomized comparison of unilateral laminotomy, bilateral laminotomy, and laminectomy. J Neurosurg Spine 2005;3:129-41.

4. Mobbs RJ, Sivabalan P, Li J. Minimally invasive surgery compared to open spinal fusion for the treatment of degenerative lumbar spine pathologies. J Clin Neurosci 2012;19:829-35.

5. Hides JA, Richardson CA, Jull GA. Multifidus muscle 
recovery is not automatic after resolution of acute, first-episode low back pain. Spine (Phila Pa 1976) 1996;21:2763-9.

6. Rahman M, Summers LE, Richter B, Mimran RI, Jacob RP. Comparison of techniques for decompressive lumbar laminectomy: the minimally invasive versus the "classic" open approach. Minim Invasive Neurosurg 2008;51:100-5.

7. Haher TR, O’Brien M, Dryer JW, Nucci R, Zipnick R, Leone DJ. The role of the lumbar facet joints in spinal stability: identification of alternative paths of loading. Spine (Phila Pa 1976) 1994;19:2667-71.

8. Schick U, Dohnert J, Richter A, Konig A, Vitzthum HE. Microendoscopic lumbar discectomy versus open surgery: an intraoperative EMG study. Eur Spine J 2002;11:20-6.

9. Weber BR, Grob D, Dvorak J, Muntener M. Posterior surgical approach to the lumbar spine and its effect on the multifidus muscle. Spine (Phila Pa 1976) 1997;22:1765-72.

10. Bassewitz H, Herkowitz H. Lumbar stenosis with spondylolisthesis: current concepts of surgical treatment. Clin Orthop Relat Res 2001;(384):54-60.

11. Fischgrund JS, Mackay M, Herkowitz HN, Brower R, Montgomery DM, Kurz LT. 1997 Volvo Award winner in clinical studies: degenerative lumbar spondylolisthesis with spinal stenosis: a prospective, randomized study comparing decompressive laminectomy and arthrodesis with and without spinal instrumentation. Spine (Phila Pa 1976) 1997;22:280712.

12. North RB, Ewend MG, Lawton MT, Kidd DH, Piantadosi S. Failed back surgery syndrome: 5-year follow-up after spinal cord stimulator implantation. Neurosurgery 1991;28:692-9.

13. Benoist M, Ficat C, Baraf P, et al. Postoperative sciatica from epidural fibrosis and lumbar arachnoiditis: results of 38 repeat operations. Rev Rhum Mal Osteoartic 1979;46:593-9.

14. Yoshimoto M, Takebayashi T, Kawaguchi S, et al. Minimally invasive technique for decompression of lumbar foraminal stenosis using a spinal microendoscope: technical note. Minim Invasive Neurosurg 2011;54:142-6.

15. Hamasaki T, Tanaka N, Kim J, Okada M, Ochi M, Hutton WC. Biomechanical assessment of minimally invasive decompression for lumbar spinal canal stenosis: a cadaver study. J Spinal Disord Tech 2009;22:486-91.

16. Bresnahan L, Fessler RG, Natarajan RN. Evaluation of change in muscle activity as a result of posterior lumbar spine surgery using a dynamic modeling system. Spine (Phila Pa 1976) 2010;35:E761-7.

17. Adams MA, Hutton WC. The mechanical function of the lumbar apophyseal joints. Spine (Phila Pa 1976) 1983;8:327-30.

18. Adams MA, Hutton WC, Stott JR. The resistance to flexion of the lumbar intervertebral joint. Spine (Phila Pa 1976) 1980;5:245-53.

19. Bresnahan L, Ogden AT, Natarajan RN, Fessler RG. A biomechanical evaluation of graded posterior element removal for treatment of lumbar stenosis: comparison of a minimally invasive approach with two standard laminectomy techniques. Spine (Phila Pa 1976) 2009;34:17-23.

20. Weiner BK, Walker M, Brower RS, McCulloch JA. Microdecompression for lumbar spinal canal stenosis. Spine (Phila Pa 1976) 1999;24:2268-72.

21. Musluman AM, Cansever T, Yilmaz A, Cavusoglu H, Yuce I, Aydin Y. Midterm outcome after a microsurgical unilateral approach for bilateral decompression of lumbar degenerative spondylolisthesis. J Neurosurg Spine 2012;16:68-76.

22. Oertel MF, Ryang YM, Korinth MC, Gilsbach JM, Rohde V. Long-term results of microsurgical treatment of lumbar spinal stenosis by unilateral laminotomy for bilateral decompression. Neurosurgery 2006;59:1264-70.

23. Kim CW. Scientific basis of minimally invasive spine surgery: prevention of multifidus muscle injury during posterior lumbar surgery. Spine (Phila Pa 1976) 2010;35(26 Suppl):S281-6.

24. Ikuta K, Arima J, Tanaka T, et al. Short-term results of microendoscopic posterior decompression for lumbar spinal stenosis: technical note. J Neurosurg Spine 2005;2:624-33.

25. Anderson DG, Patel A, Maltenfort M, et al. Lumbar decompression using a traditional midline approach versus a tubular retractor system: comparison of patient-based clinical outcomes. Spine (Phila Pa 1976) 2011;36:E320-5.

26. Kambin, P. Percutaneous lumbar discectomy (Triangular Working Zone): current practice. Surg Rounds Orthop1988:31-35. 
27. Ruetten S, Komp M, Merk H, Godolias G. Surgical treatment for lumbar lateral recess stenosis with the full-endoscopic interlaminar approach versus conventional microsurgical technique: a prospective, randomized, controlled study. J Neurosurg Spine 2009;10:476-85.

28. Komp M, Hahn P, Merk H, Godolias G, Ruetten S. Bilateral operation of lumbar degenerative central spinal stenosis in full-endoscopic interlaminar technique with unilateral approach: prospective 2-year results of 74 patients. J Spinal Disord Tech 2011;24:281-7.

29. Caputy AJ, Luessenhop AJ. Long-term evaluation of decompressive surgery for degenerative lumbar stenosis. J Neurosurg 1992;77:669-76.

30. Mayer HM, List J, Korge A, Wiechert K. Microsurgery of acquired degenerative lumbar spinal stenosis. Bilateral over-the-top decompression through unilateral approach. Orthopade 2003;32:889-95. 